\title{
Probing tricarbocyanine dyes for targeted delivery of anthracyclines
}

\author{
Dmitriy A. Veriutinn ${ }^{\mathrm{a}, \mathrm{b}, \mathrm{c}}$, Irina A. Doroshenko ${ }^{\mathrm{a}}$, Ekaterina A. Martynova ${ }^{\mathrm{a}}$, Ksenya A. Sapozhnikova ${ }^{\mathrm{c}}$, \\ Elena V. Svirshchevskaya ${ }^{\mathrm{c}}$, Anna V. Shibaeva ${ }^{\mathrm{e}}$, Alina A. Markova ${ }^{\mathrm{e}, \mathrm{f}}$, Alexey A. Chistov ${ }^{\mathrm{c}, \mathrm{d}}$, \\ Natalya E. Borisova ${ }^{\mathrm{a}}$, Maxim V. Shuvalov ${ }^{\mathrm{a}, \mathrm{b}}$, Vladimir A. Korshun ${ }^{\mathrm{b}, \mathrm{c}}$, Vera A. Alferova ${ }^{\mathrm{b}, \mathrm{c}, * *}$ and \\ Tatyana A. Podrugina ${ }^{\mathrm{a}, *}$
}

${ }^{a}$ Lomonosov Moscow State University, Department of Chemistry, Leninskiye Gory 1, Moscow 119992, Russia

${ }^{b}$ Gause Institute of New Antibiotics, B. Pirogovskaya 11, Moscow 119021, Russia

${ }^{c}$ Shemyakin-Ovchinnikov Institute of Bioorganic Chemistry, Miklukho-Maklaya 16/10, Moscow 117997, Russia

${ }^{d}$ Orekhovich Research Institute of Biomedical Chemistry, Pogodinskaya 10, Moscow 119121, Russia

${ }^{e}$ Emanuel Institute of Biochemical Physics, Kosygina 4, Moscow 119334, Russia

${ }^{f}$ Nesmeyanov Institute of Organoelement Compounds, Vavilova 28, Moscow 119991, Russia

\section{ABSTRACT}

Keywords:

Tricarbocyanine dyes

Anthracyclines

Drug conjugates

Cytotoxicity

ROS generation
Heptamethine carbocyanine dyes possess bright fluorescence in the near IR range and affinity to cancer cells. Thus, these dyes could be utilized as fluorescent labels and vectors for drug delivery in their covalent conjugates with cytotoxic compounds. In this work we synthesized four drugdye conjugates of tricarbocyanine dyes with anthracycline drug daunorubicin using a CuAAC reaction. Conjugates with hydrophobic dyes possess submicromolar cytotoxicity. Fluorescent imaging revealed significant accumulation of the conjugates in mitochondria, suggesting an enhancement of an additional mechanism of anthracycline cytotoxicity - generation of ROS. The hypothesis was supported by significant reduction of activity of the conjugates in presence of an antioxidant compound.

\section{Introduction}

Heptamethine carbocyanine (tricarbocyanine) dyes (Figure 1) possess outstanding photophysical properties, namely, high molar absorption and emission coefficients and a narrow absorption/emission band. Their fluorescent maxima are located in the near infrared (NIR) range (700-1000 nm, NIR-I window), where biological tissues are transparent, ${ }^{1}$ thus making them suitable for bioimaging. ${ }^{2,3}$ Moreover, most common photodetectors have sensitivity up to $900 \mathrm{~nm},{ }^{4}$ thus making NIRI dyes convenient for widely used bioimaging equipment.<smiles>[R]C1=C(/C=C/C2=[N+]([R1])CCC([R])C2)CCC/C1=C\C=C1/CC([R8])CN1[R]</smiles>

Figure 1. A general structure of tricarbocyanine dyes. $R^{1}-R^{5}-$ variable substituents.

The unique photophysical properties of these dyes make them attractive as photosensitizers for photodynamic/photothermal therapy, ${ }^{5-7}$ including nanoparticle conjugates. ${ }^{8-10}$ Besides, these dyes have been applied as light- or hydrolysis-mediated photocages for controlled release of therapeutic molecules, for

\footnotetext{
* Corresponding author.
}

** Corresponding author.

E-mail addresses: alferovava@gmail.com (V.A. Alferova), podrugina@mail.ru (T.A. Podrugina). example for cyclosporine $\mathrm{A},{ }^{11} \quad$ camptothecin $^{12}$ and chlorambucil. ${ }^{13}$

Another remarkable feature of tricarbocyanines is their preferential accumulation and retention in tumour tissues. ${ }^{14} \mathrm{~A}$ proposed explanation of this phenomenon includes several factors ${ }^{15}$ such as hypoxia-mediated overexpression of organic anion transporting polypeptides (OATPs), ${ }^{16}$ greater negative mitochondrial membrane potential in cancer cells compared to normal ones ${ }^{17}$ and formation of covalent adducts with biomolecules, like albumin. ${ }^{18}$

Abovementioned properties of tricarbocyanines were utilized for the development of fluorescent and theranostic conjugates. ${ }^{2,14}$ Carbocyanine dyes were applied as delivery vectors in covalent conjugates with various anticancer agents and in some cases were shown to increase their cytotoxicity. ${ }^{2,14,19}$ For example, conjugate of non-toxic cytoprotective agent troxipide with dye MHI-148 showed nanomolar cytotoxic activity $\left(\mathrm{EC}_{50} 267 \mathrm{nM}\right)$ on three glioblastoma cell lines. ${ }^{20}$ The same dye linked with the renin angiotensin system (Ras) inhibitor S-trans-trans farnesylthiosalicylic acid (FTS) significantly improved specific aggregation of this acid in pancreatic tumor cells. ${ }^{21}$ Another important example is the conjugate of tricarbocyanine dye with oxoplatin, showing micromolar activity against Burkitt lymphoma cells and accumulating specificially in the tumor area in mice. ${ }^{22}$ Mitosis inhibitor cabazitaxel linked with a 
carbocyanine dye exhibited micromolar cytotoxicity against drug-resistant renal cell carcinoma cells, which is superior to that of the native dye and drug. ${ }^{23}$ Some conjugates of kinase inhibitor erlotinib with heptamethine carbocyanines have shown up to 50-fold increase in cytotoxicity, compared to initial erlotinib. ${ }^{24}$ Moreover, conjugation of carbocyanine dye IR-783 with flavonoid-based natural compound genistein led to 2-fold lower $\mathrm{IC}_{50}$ than that of the non-conjugated drug. ${ }^{25}$

These examples indicate the high potential of covalent conjugates comprised of a cytotoxic compound and a carbocyanine dye, for drug development. Nonetheless, there are very few $^{26}$ examples of covalent conjugates of dyes with representatives one of the most effective anticancer drugs families - anthracyclines. These drugs are widely used in clinica practice to treat different types of cancer, e.g. leukemia, lymphoma, lung cancer etc. ${ }^{27}$ Their cytotoxicity mechanism is complex, but the most pronounced mode of action in clinical concentrations is topoisomerase II $\alpha$ inhibition. ${ }^{28}$ Besides their high cytotoxicity, anthracyclines have serious side effects, the most severe is potentially lethal cumulative cardiotoxity. ${ }^{29}$ Side effects of anthracyclines can be reduced if they are used in tumor targeting systems, e.g. liposomes, ${ }^{30}$ nanoparticles ${ }^{31}$ or covalent conjugates with delivery vectors.

In this work we report the synthesis of conjugates based on heptamethine cyanine dyes and anthracycline antibiotic daunorubicin. Conjugation was performed by $\mathrm{Cu}$-catalysed azide-alkyne cycloaddition (CuAAC), one of the so-called "click reactions". These reactions are very suitable for labile compounds, because of their high selectivity, almost quantitative yields and mild conditions required. ${ }^{32}$

\section{Results and discussion}

\subsection{Synthesis}

To synthesize the conjugates by click-reaction, we first had to introduce a suitable functional group into the daunorubicin molecule. There is a number of previously known approaches to modification of anthracycline antibiotics (Figure 2). In this work we introduced the azide group in a daunorubicin via acylation of the 3 '-amino group (site a) ${ }^{33}$ and via synthesis of an oxime derivative on the 13-carbonyl group (site d). ${ }^{34}$

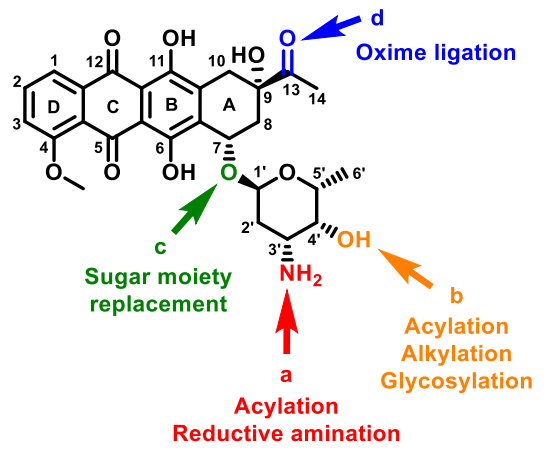

Figure 2. Known sites for modification of daunorubicin..$^{27,33,34}$

Azide derivative 1 (Figure 3) was synthesized by acylation of the 3 -amino group of daunorubicin with commercially available oxysuccinimide 4-azidobutanoate using a previously reported procedure. ${ }^{35}$ The resulting compound $\mathbf{1}$ was obtained with high yield $(83 \%)$. For compound 2, oxime ligation with $O$-(2-(2azidoethoxy)ethyl)-hydroxylammonium chloride ${ }^{36}$ was used (Scheme 1). The synthesis was conducted on the basis of procedure for oxime ligation of olivomicin ${ }^{37}$ with some alterations: reduced oxyamine excess from 5 to 3 -fold, DMSO as the solvent, no pyridine treatment, and addition of dry $\mathrm{Na}_{2} \mathrm{SO}_{4}$ into the reaction mixture for water removal. The resulting compound 3 was obtained with high yield (82\%).

The second task was the synthesis of indocyanine dyes bearing a terminal alkyne moiety suitable for conjugation with daunorubicin derivatives $\mathbf{1}$ and $\boldsymbol{2}$. We obtained two unsymmetrical dyes, containing a terminal alkyne moiety in the quaternary nitrogen atom (5a-b, Scheme 2$)$. Their synthesis was based on known general procedures. ${ }^{38-41} \mathrm{~N}$-alkylated indolenine salt 3a-b was coupled with $\mathrm{N}-((1 E, 2 E, 4 E)-5$ (phenylamino)penta-2,4-dien-1-ylidene)benzenaminium chloride followed by condensation with 2-methyl-3-(prop-2-yn1-yl)benzo[d]thiazol-3-ium under basic conditions to yield unsymmetrical tricarbocyanine dyes $\mathbf{5 a}$ and $\mathbf{5 b}$, respectively.

Based on dyes $\mathbf{3 c}$ and $\mathbf{4 c}$ (Figure 4), four novel daunorubicin conjugates were synthesized via a CuAAC reaction $(\mathbf{6 a - b}, \mathbf{7 a - b}$, Scheme 3). ${ }^{32}$

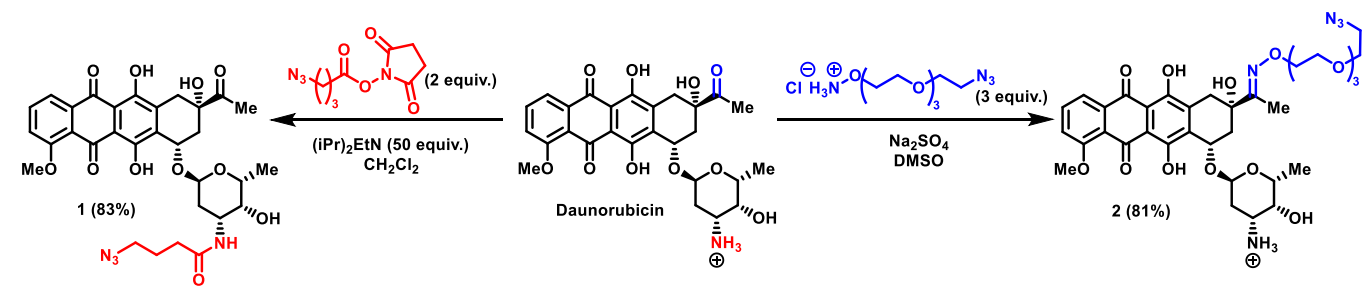

Scheme 1. Synthesis of alkylazide daunorubicin derivatives $\mathbf{1}$ and $\mathbf{2}$.

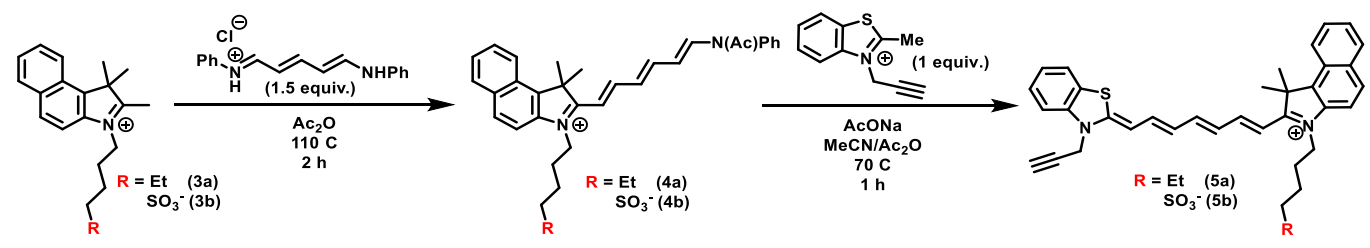

Scheme 2. Synthesis of functionalized tricarbocyanine dyes used in this work. 
The compositions of the conjugates were confirmed by massspectra. UV/Vis absorption spectra contained the characteristic signals at 480 and $780-790 \mathrm{~nm}$. According to absorption spectra, the molar ratio between anthracycline and dye chromophores is 1:1 for all the obtained conjugates. Conjugates exhibit emission bands characteristic for both anthracycline $(586 \mathrm{~nm})$ and tricarbocyanine $(818-833 \mathrm{~nm})$.

\subsection{Biological evaluation}

Cytotoxicity of the obtained compounds was measured by MTT-test on 4 cell lines: murine colon carcinoma CT26, human metastatic pancreatic adenocarcinoma COLO357, pseudonormal tumorigenic in nude mice human breast epithelial cells HBL100 and human colon cancer cells HCT116 (Table 1).

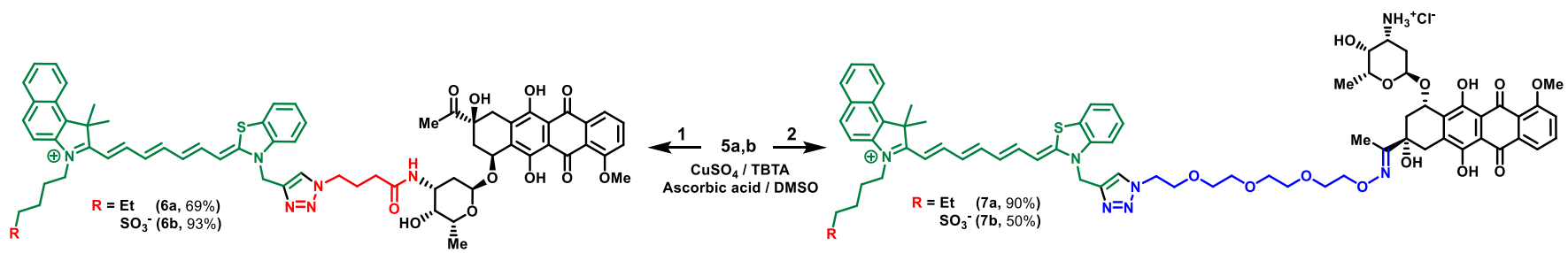

Scheme 3. Synthesis of conjugates $\mathbf{6 a - b}$ and $7 \mathbf{a}-\mathbf{b}$.

Table 1. Cytotoxicity of the compounds obtained.

\begin{tabular}{ccccc}
\hline \multirow{2}{*}{ Compound } & \multicolumn{4}{c}{$\mathrm{IC}_{50}, \mu \mathrm{M}$} \\
\cline { 2 - 5 } & $\mathrm{CT26}$ & $\mathrm{COLO} 357$ & $\mathrm{HBL} 100$ & $\mathrm{HCT116}$ \\
\hline Daunorubicin & 0.032 & $<0.032$ & $<0.032$ & 1 \\
$\mathbf{1}$ & 1 & 0.8 & 4 & 1 \\
$\mathbf{2}$ & 4 & 4 & 4 & 1 \\
$\mathbf{5 a}$ & 0.2 & 1 & 1 & 1 \\
$\mathbf{5 b}$ & $>100$ & $>100$ & $>100$ & 18 \\
$\mathbf{6 a}$ & 20 & 4 & 20 & 3 \\
$\mathbf{6 b}$ & $>100$ & $>100$ & $>100$ & $>100$ \\
$\mathbf{7 a}$ & 4 & 2 & 1 & 1 \\
$\mathbf{7 b}$ & $>100$ & $>100$ & $>100$ & $>100$ \\
\hline
\end{tabular}

According to literature, ${ }^{33}$ acylation of the 3 -amino group significantly decreases cytotoxicity of anthracyclines, impairing their ability to penetrate the cell membrane. Surprisingly, daunorubicin derivative $\mathbf{1}$ with the acylated amino group was found to be more active than $\mathbf{2}$. However, cytotoxicity of both derivatives (1 and $\mathbf{2}$ ), as well as of conjugates with lipophilic dyes

Daunorubicin
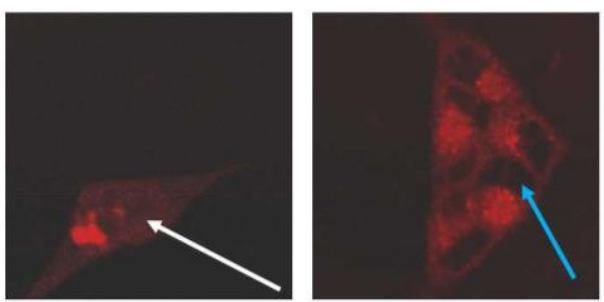

(6a, 7a), was lower than that of the parent drug. Conjugates with sulfo-tricarbocyanines $(\mathbf{6 b}, \mathbf{7 b})$ displayed no activity, presumably due to their impaired transportability in membranes. It was recently shown that polar negatively charged groups could decrease cytotoxicity of carbocyanine-drug conjugates. ${ }^{24}$ The same trend is observed for the dyes, $\mathbf{5 b}$ has no cytotoxicity because of its inability to get through the cell membrane. ${ }^{42}$

Anticancer agents relying on direct nuclear DNA damage could make stable mutations in normal cells and this way induce tumor recurrence, a very dangerous side-effect. ${ }^{43}$ Mitochondriatargeting is one of the prospective approaches to overcome this problem. ${ }^{43}$ Tricarbocyanine dyes are able to accumulate in mitochondria; ${ }^{44}$ this allows us to suggest that our conjugates possess the same property. The accumulation of the conjugates in the cells was visualized with confocal microscopy (Figure 3). Compared to daunorubicin and the known antitumor anthracycline, doxorubicin, compound $\mathbf{7 a}$ is distributed in the cytoplasm of the cell and is not visualized in the nucleus (blue arrow shows unstained nuclei, white arrows show nuclei with anthracycline fluorescence signal).

\section{Doxorubicin}

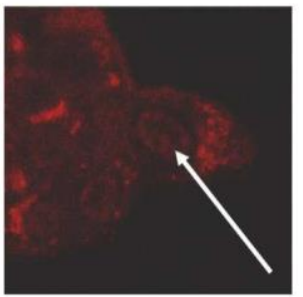

Figure 3. Confocal imaging of HCT116 cells treated with the conjugate 7a, daunorubicin and doxorubicin $(24 \mathrm{~h}, 1 \mu \mathrm{M})$. The compound 7a was detected with excitation at $633 \mathrm{~nm}$ and emission range 699-800 $\mathrm{nm}$ (daunorubicin and doxorubicin: Ex $488 \mathrm{Em} 500-650 \mathrm{~nm}$ ).

The results of colocalization with lysotracker and DHR123 show that conjugate 7a accumulated preferentially in mitochondria and less in lysosomes of the treated cells (Figure 4). 


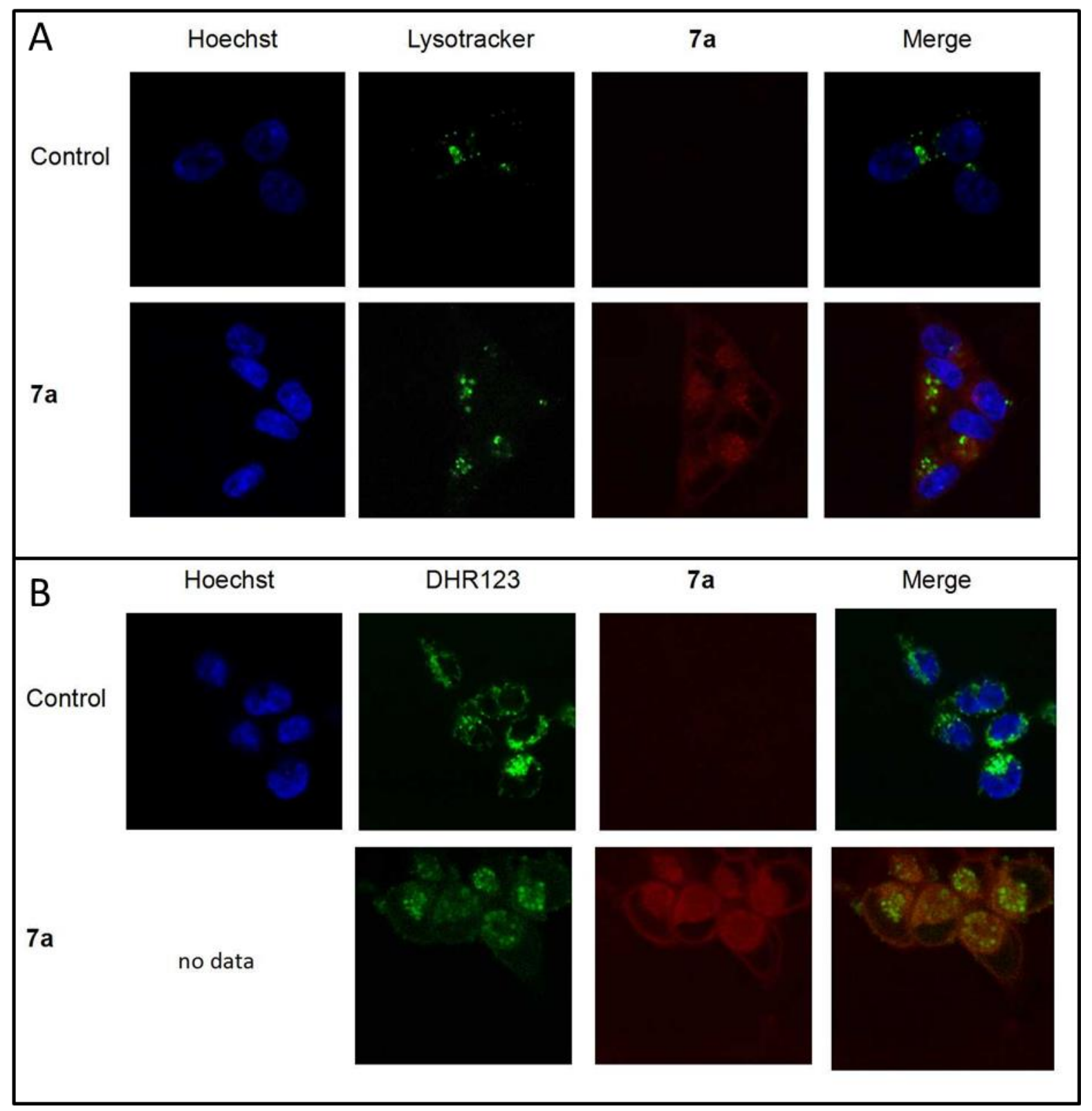

Figure 4. Confocal imaging of HCT116 cells, treated with the conjugate 7a $(24 \mathrm{~h}, 1 \mu \mathrm{M})$, dyed with Hoechst and Lysotracker (A) and DHR123 (B). 7a: Ex 633 Em 699-800 nm; Lysotracker: Ex 476 Em 509-582 nm; DHR123: Ex 476 Em 509-582 nm; Hoechst: Ex 405 Em $410-490$ nm.

In addition to the predominant distribution of the 7a derivative in mitochondria, their significant damage is seen in comparison with the control (cells untreated with anthracycline) mitochondria acquired a spherical shape and significantly increased in size. DHR123 has been used as a mitochondrial tracker to significantly increase intrinsic fluorescence in the presence of ROS.

While the both components of the conjugate are capable of generating ROS, delivery to the mitochondria might lead to the increase of the contribution of this mechanism to the overall cytotoxic effect. Therefore, we evaluated cytotoxicity of the conjugates in presence of antioxidant NAC $100 \mu \mathrm{M}$ with preincubation $12 \mathrm{~h}$ before adding $7 \mathbf{a}$ (Figure 5).

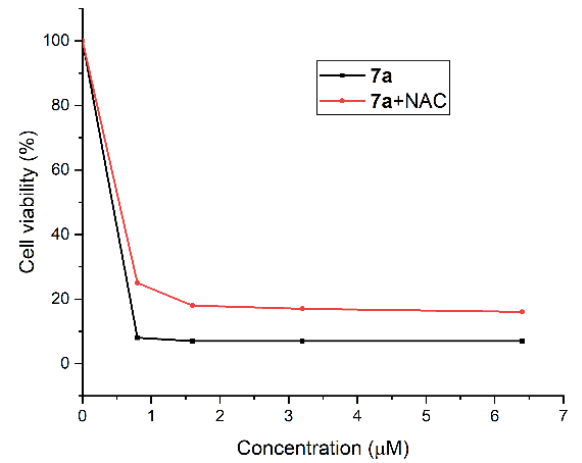

Figure 5. MTT-assay for HCT116 cells, treated with the conjugate 7a (96h, $0.8-6.4 \mu \mathrm{M}$ ) in the presence and absence of NAC (after 5 independent repeats, errors did not exceed $10 \%$ ). 
Dose-dependent cell death in the presence of NAC is less pronounced than without the antioxidant. This allows us to conclude that the mechanism of cell death under the action of derivative $\mathbf{7 a}$ is associated with the generation of ROS.

\section{Conclusions}

To conclude, we synthesized a series of tricarbocyanineanthracycline conjugates and evaluated their cytotoxic properties. Hydrophobic dyes were found to be capable of selective delivery of the anthracycline to the mitochondria, leading to significant contribution of ROS generation to the mechanism of action of the conjugates. Cytotoxicity of the covalent conjugates was found to be lower than that of the parent drug. Nonetheless, structure optimization might overcome this problem, thus making the conjugates of tricarbocyanines and anthracyclines an attractive target for further drug development.

\section{Experimental section}

\subsection{Materials and methods}

Thin-layer chromatography was performed using Merck silica gel $60 \mathrm{~F}_{254}$ plates. For column chromatography, Merck Silica gel 60 was used. NMR experiments were carried out on Bruker Avance 400 and Bruker Avance II 600 spectrometers. Routine ESI LCMS mass spectra were acquired using Agilent 6340 Ion Trap equipped with an electrospray ionization source (ESI) ("Agilent Technologies") coupled to an Agilent 1100 HPLC system. ESI HRMS spectra were recorded using Bruker micrOTOF II system. Analytical HPLC was performed using a Knauer HPLC system equipped with a K-2501 detector, using Sunfire C18 $(5 \mu \mathrm{m}$, $4.6 \times 250 \mathrm{~mm})$ and VDSphere PUR $100 \mathrm{C} 8$-E columns $(7 \mu \mathrm{m}$, $46 \times 250 \mathrm{~mm}$ ). UV-Vis absorption spectra were recorded using a Varian Cary $100 \mathrm{UV}-\mathrm{V}$ is spectrophotometer. The fluorescence studies were carried out on a Perkin-Elmer LS 55 luminescence spectrometer. Freeze-drying was performed using FreeZone 1 Liter Benchtop Freezy Dry Systems. Evaporation under reduced pressure was performed with Labconco Acid-Resistant CentriVap Centrifugal Vacuum Concentrator and Heidolph Hei-VAP Precision rotary evaporator. The solvents used in this work were of the highest grade available.

$\mathrm{N}-((1 E, 3 E, 5 E)-5-($ phenylimino)penta-1,3-dien-1-yl)aniline hydrochloride ${ }^{39}$, compound $\mathbf{4 a},{ }^{40}$ and 2-methyl-3-(prop-2-yn-1yl)benzo[d]thiazol-3-ium bromide ${ }^{41}$ were synthesized according to literature procedures. Compound $\mathbf{4 b}$ was synthesized similarly to the compound $\mathbf{4 a}$.

\subsection{Synthesis of 3-hexyl-1,1,2-trimethyl-1H-benzo[e]indol-3-ium iodide (3a)}

The procedure was based on ${ }^{38}$ with some modifications. 1,1,2Trimethyl- $1 \mathrm{H}$-benzo[ $e$ ]indole $(0.50 \mathrm{~g}, 2.4 \mathrm{mmol})$ and 1iodohexane $(1.3 \mathrm{~mL}, 7.2 \mathrm{mmol})$ in acetonitrile $(5 \mathrm{~mL})$ were stirred upon heating to $72^{\circ} \mathrm{C}$ for $24 \mathrm{~h}$. After cooling to room temperature, an excess of diethyl ether was added, the precipitate was filtered off, washed by diethyl ether and dried. Yield: $0.78 \mathrm{~g} \mathrm{(88 \% ),} \mathrm{light}$ gray powder. M.p. $160^{\circ} \mathrm{C} .{ }^{1} \mathrm{H}$ NMR spectrum (DMSO- $d_{6}, \delta$, ppm, $J / \mathrm{Hz}$ ): 0.84-0.89 (m, $3 \mathrm{H}, \mathrm{CH}_{3}$ ), 1.31 (br. s., $4 \mathrm{H}, 2 \mathrm{CH}_{2}$ ), 1.46 (br. s., $2 \mathrm{H}, \mathrm{CH}_{2}$ ), 1.76 (s, $\left.6 \mathrm{H}, \mathrm{C}\left(\mathrm{CH}_{3}\right)_{2}\right), 1.89$ (br. s., $2 \mathrm{H}, \mathrm{CH}_{2}$ ), 2.95 $\left(\mathrm{s}, 3 \mathrm{H},=\mathrm{C}-\mathrm{CH}_{3}\right), 4.58\left(\mathrm{t}, 2 \mathrm{H},{ }^{3} \mathrm{~J}_{\mathrm{HH}}=7.2, \mathrm{CH}_{2} \mathrm{~N}+\right), 7.70-7.82(\mathrm{~m}$, $2 \mathrm{H}$, aromatic), $8.16\left(\mathrm{~d}, 1 \mathrm{H},{ }^{3} \mathrm{~J}_{\mathrm{HH}}=9.0\right.$, aromatic $), 8.22(\mathrm{~d}, 1 \mathrm{H}$, ${ }^{3} J_{\mathrm{HH}}=8.1$, aromatic $), 8.30\left(\mathrm{~d}, 1 \mathrm{H},{ }^{3} J_{\mathrm{HH}}=8.8\right.$, aromatic $), 8.38(\mathrm{~d}$, $1 \mathrm{H},{ }^{3} J_{\mathrm{HH}}=8.2$, aromatic). ${ }^{13} \mathrm{C}$ NMR spectrum (DMSO- $d_{6}, \delta$, ppm): 13.6 (s, $\left.1 \mathrm{C}, \underline{\mathrm{CH}}_{3}\right), 13.8\left(\mathrm{~s}, 1 \mathrm{C},=\mathrm{C}-\underline{\mathrm{CH}}_{3}\right), 21.4(\mathrm{~s}, 2 \mathrm{C}$, $\left.\mathrm{C}\left(\mathrm{CH}_{3}\right)_{2}\right), 21.7,25.3,27.2,30.6$ (all s, $1 \mathrm{C}$, all $\left.\mathrm{CH}_{2}\right), 47.7$ (s, $1 \mathrm{C}$, $\left.\mathrm{CH}_{2} \mathrm{~N}+\right), 55.3$ (s, $\left.1 \mathrm{C}, \underline{\mathrm{C}}\left(\mathrm{CH}_{3}\right)_{2}\right), 113.1,123.2,126.9,1297.0$, $128.2,129.5,130.5,132.8,136.7,138.2$ (all s, $1 \mathrm{C}$, all aromatic),
196.0 (s, $1 \mathrm{C},=\mathrm{C}-\mathrm{CH}_{3}$ ). HRMS-ESI: found: $\mathrm{m} / \mathrm{z}, 294.2217 \mathrm{M}$. $\mathrm{C}_{21} \mathrm{H}_{28} \mathrm{~N}$. Calculated: $\mathrm{M}=294.2216$. IR, $v / \mathrm{cm}^{-1}$ : $1130(\mathrm{C}-\mathrm{N}$, tetr.am.), $1466\left(\mathrm{CH}_{2}, \mathrm{CH}_{3}\right)$

\subsection{Synthesis of 4-(1,1,2-trimethyl-1H-benzo[e]indol-3-ium-3- yl)butane-1-sulfonate $(\mathbf{3 b})$}

Procedure was based on ${ }^{45}$ with some modifications. A mixture of 1,1,2-trimethyl-1 $H$-benzo[ $e$ ]indole $(0.78 \mathrm{~g}, 3.73 \mathrm{mmol})$ and 1,4 butane sultone $(0.40 \mathrm{~mL}, 4.02 \mathrm{mmol})$ in $o$-diclorobenzene $(3.0$ $\mathrm{mL}$ ) was stirred under $120^{\circ} \mathrm{C}$ for $8 \mathrm{~h}$. After cooling to room temperature, an excess of diethyl ether was added; the precipitate formed was filtered off, washed by diethyl ether and dried. Yield: $0.75 \mathrm{~g}(59 \%)$, light blue powder. Dec. $>200^{\circ} \mathrm{C}$. ${ }^{1} \mathrm{H}$ NMR spectrum (DMSO- $\left.d_{6}, \delta, \mathrm{ppm}, J / \mathrm{Hz}\right): 1.70-1.82\left(\mathrm{~m}, 8 \mathrm{H}, \mathrm{CH}_{2}, \mathrm{C}\left(\mathrm{CH}_{3}\right)_{2}\right), 2.02$ (br.s., $2 \mathrm{H}, \mathrm{CH}_{2}$ ), 2.52-2.56 (m, $2 \mathrm{H}, \mathrm{CH}_{2} \mathrm{SO}_{3}$ ), 2.95 (br.s., $3 \mathrm{H}$, $\left.=\mathrm{C}-\mathrm{CH}_{3}\right), 4.61$ (br.s., $\left.2 \mathrm{H}, \mathrm{CH}_{2} \mathrm{~N}+\right), 7.66-7.82(\mathrm{~m}, 2 \mathrm{H}$, aromatic), $8.16-8.30\left(\mathrm{~m}, 3 \mathrm{H}\right.$, aromatic), $8.36\left(\mathrm{~d}, 1 \mathrm{H},{ }^{3} J_{\mathrm{HH}}=7.5\right.$, aromatic $)$.

4.4. Synthesis of 3-hexyl-1,1-dimethyl-2-((1E,3E,5E,7Z)-7-(3(prop-2-yn-1-yl)benzo[d] thiazol-2(3H)-ylidene)hepta-1,3,5-trien1-yl)-1H-benzo[e]indol-3-ium iodide (5a)

Mixture of 3-hexyl-1,1-dimethyl-2-((1E,3E,5E)-6-(Nphenylacetamido)hexa-1,3,5-trien-1-yl)-1H-benzo[e]indol-3-ium iodide (4a) (0.10 g, $0.16 \mathrm{mmol})$, 2-methyl-3-(prop-2-yn-1yl)benzo $[d]$ thiazol-3-ium bromide $(0.040 \mathrm{~g}, 0.16 \mathrm{mmol})$, and sodium acetate $(0.030 \mathrm{~g}, 0.32 \mathrm{mmol})$ of in acetonitrile $(2.0 \mathrm{~mL})$ was stirred under $70^{\circ} \mathrm{C}$ for $4 \mathrm{~h}$. After cooling to room temperature, an excess of diethyl ether was added, the precipitate formed was filtered off, washed by diethyl ether and dried. Then the dye was purified by silica gel column chromatography (elution with $\mathrm{CH}_{2} \mathrm{Cl}_{2}-\mathrm{MeOH}$, 50:1) followed by silica gel thin-layer chromatography (eluent $\mathrm{CH}_{2} \mathrm{Cl}_{2}-\mathrm{MeOH}, 10: 1$ ). Yield: $0.012 \mathrm{~g}$ $(11 \%)$, dark green powder. ${ }^{1} \mathrm{H}$ NMR spectrum $\left(\mathrm{CDCl}_{3}, \delta\right.$, ppm, $\mathrm{J} / \mathrm{Hz}): 0.91\left(\mathrm{t}, 3 \mathrm{H},{ }^{3} \mathrm{~J}_{\mathrm{HH}}=7.2, \mathrm{CH}_{3}\right), 1.35-1.38\left(\mathrm{~m}, 4 \mathrm{H}, 2 \mathrm{CH}_{2}\right)$, 1.47-1.50 (m, $\left.2 \mathrm{H}, \mathrm{CH}_{2}\right), 1.82-1.86\left(\mathrm{~m}, 2 \mathrm{H}, \mathrm{CH}_{2}\right), 2.00(\mathrm{~s}, 6 \mathrm{H}$, $\left.\mathrm{C}\left(\mathrm{CH}_{3}\right)_{2}\right), 2.47$ (br.s., $\left.1 \mathrm{H}, \mathrm{C} \equiv \mathrm{CH}\right), 4.08$ (t, $2 \mathrm{H},{ }^{3} J_{\mathrm{HH}}=7.3$, $+\mathrm{NCH}_{2}$ ), 5.25 (br. s., $\left.2 \mathrm{H}, \underline{\mathrm{CH}}_{2} \mathrm{C} \equiv \mathrm{CH}\right), 6.13\left(\mathrm{~d}, 1 \mathrm{H},{ }^{3} \mathrm{~J}_{\mathrm{HH}}=13.1\right.$, $=\mathrm{CH}), 6.57\left(\mathrm{t}, 1 \mathrm{H},{ }^{3} J_{\mathrm{HH}}=12.3,=\mathrm{CH}\right), 7.28-7.32(\mathrm{~m}, 2 \mathrm{H}, 2=\mathrm{CH})$, $7.37\left(\mathrm{t}, 1 \mathrm{H},{ }^{3} J_{\mathrm{HH}}=7.3\right.$, aromatic $), 7.45\left(\mathrm{t}, 2 \mathrm{H},{ }^{3} J_{\mathrm{HH}}=7.5\right.$, aromatic), 7.49-7.55 (m, $3 \mathrm{H}$, aromatic, $2=\mathrm{CH}), 7.60\left(\mathrm{t}, 2 \mathrm{H},{ }^{3} J_{\mathrm{HH}}\right.$ = 7.5, aromatic), $7.78\left(\mathrm{~d}, 1 \mathrm{H},{ }^{3} \mathrm{~J}_{\mathrm{HH}}=7.3\right.$, aromatic $), 7.89-7.94(\mathrm{~m}$, $3 \mathrm{H}$, aromatic, $=\mathrm{CH}), 8.12\left(\mathrm{~d}, 1 \mathrm{H},{ }^{3} \mathrm{~J}_{\mathrm{HH}}=8.6\right.$, aromatic $) .{ }^{13} \mathrm{C} \mathrm{NMR}$ spectrum $\left(\mathrm{CDCl}_{3}, \delta, \mathrm{ppm}, \mathrm{J} / \mathrm{Hz}\right): 13.9\left(\mathrm{~s}, 1 \mathrm{C}, \mathrm{CH}_{3}\right), 22.45,26.6$ (all s, $1 \mathrm{C}$, all $\left.\mathrm{CH}_{2}\right), 27.7$ (s, $\left.2 \mathrm{C}, \mathrm{C}\left(\underline{\mathrm{CH}}_{3}\right)_{2}\right), 29.7,31.5$ (all s, $1 \mathrm{C}$,

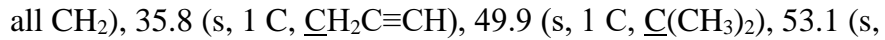
$\left.1 \mathrm{C},+\mathrm{NCH}_{2}\right), 73.4(\mathrm{~s}, 1 \mathrm{C}, \mathrm{C} \equiv \underline{\mathrm{C}} \mathrm{H}), 76.1(\mathrm{~s}, 1 \mathrm{C}, \underline{\mathrm{C}} \equiv \mathrm{CH}), 102.6$, $109.1($ all s, $1 \mathrm{C}$, all $=\mathrm{CH}), 110.2(\mathrm{~s}, 1 \mathrm{C}$, aromatic $), 112.8(\mathrm{~s}, 1 \mathrm{C}$, $=\mathrm{CH}), 117.5,121.2($ all s, $1 \mathrm{C}$, all aromatic), $122.2(\mathrm{~s}, 1 \mathrm{C},=\mathrm{CH})$, 122.3, 124.7, 125.6, 127.7, 128.2, 128.3 (all s, $1 \mathrm{C}$, all aromatic), $129.8(\mathrm{~s}, 1 \mathrm{C},=\mathrm{CH}), 129.9,130.4,132.0,134.1,136.4,138.8$ (all s, $1 \mathrm{C}$, all aromatic), 139.9 (s, 1 C, N-C-S), 142.7 (s, 1 C, aromatic), 151.8, $158.0($ all s, $1 \mathrm{C}$, all $=\mathrm{CH}), 192.1(\mathrm{~s}, 1 \mathrm{C},+\mathrm{N}=\mathrm{C})$. HRMSESI: found $\mathrm{m} / \mathrm{z} 543.2820[\mathrm{M}+]$. Calculated for $\mathrm{C}_{37} \mathrm{H}_{39} \mathrm{~N}_{2} \mathrm{~S}^{+}$ 543.2828. IR, $v / \mathrm{cm}^{-1}: 1360$ (N tret.), $2115(\mathrm{C} \equiv \mathrm{CH})$.

4.5. Synthesis of 4-(1,1-dimethyl-2-((1E,3E,5E,7Z)-7-(3-(prop-2yn-1-yl)benzo[d] thiazol-2(3H)-ylidene)hepta-1,3,5-trien-1-yl)1 H-benzo[e]indol-3-ium-3-yl)butane-1-sulfonate $(\mathbf{5 b})$

A mixture of 4-(1,1-dimethyl-2-((1E,3E,5E)-6-(Nphenylacetamido)hexa-1,3,5-trien-1-yl)-1H-benzo[ $e$ indol-3-ium3-yl)butane-1-sulfonate (4b) (0.10 g, $0.2 \mathrm{mmol})$, 2-methyl-3(prop-2-yn-1-yl)benzo[d]thiazol-3-ium bromide (0.03 g, 0.4 $\mathrm{mmol})$, and sodium acetate $(0.050 \mathrm{~g}, 0.2 \mathrm{mmol})$ in acetonitrile $(2$ $\mathrm{mL}$ ) was stirred at $70^{\circ} \mathrm{C}$ for $1 \mathrm{~h}$. After cooling to room temperature, an excess of diethyl ether was added, the precipitate was filtered 
off, washed by diethyl ether and dried. Further purification was performed by silica gel column chromatography (elution with $\left.\mathrm{CH}_{2} \mathrm{Cl}_{2}-\mathrm{MeOH}, 7: 1\right)$. Yield $0.025 \mathrm{~g}(21 \%)$, dark green powder. $\lambda_{\max }=775 \mathrm{~nm}(\mathrm{MeOH}) .{ }^{1} \mathrm{H}$ NMR spectrum (DMSO- $d_{6}, \delta, \mathrm{ppm}$, $\mathrm{J} / \mathrm{Hz}): 1.73-1.79\left(\mathrm{~m}, 2 \mathrm{H}, \mathrm{CH}_{2}\right), 1.80-1.85\left(\mathrm{~m}, 2 \mathrm{H}, \mathrm{CH}_{2}\right), 1.89$ (s, $\left.6 \mathrm{H}, \mathrm{C}\left(\mathrm{CH}_{3}\right)_{2}\right), 2.52$ (br.s., $\left.2 \mathrm{H}, \mathrm{CH}_{2} \mathrm{SO}_{3}\right), 3.58\left(\mathrm{t}, 1 \mathrm{H},{ }^{4} J_{\mathrm{HH}}=2.4\right.$, $\mathrm{C} \equiv \mathrm{CH}), 4.18\left(\mathrm{t}, 2 \mathrm{H},{ }^{3} J_{\mathrm{HH}}=7.5,+\mathrm{NCH}_{2}\right), 5.30\left(\mathrm{~d}, 2 \mathrm{H},{ }^{4} J_{\mathrm{HH}}=2.0\right.$, $\left.\mathrm{CH}_{2} \mathrm{C} \equiv \mathrm{CH}\right), 6.44\left(\mathrm{~d}, 1 \mathrm{H},{ }^{3} \mathrm{~J}_{\mathrm{HH}}=14.2,=\mathrm{CH}\right), 6.52\left(\mathrm{t}, 1 \mathrm{H},{ }^{3} \mathrm{~J}_{\mathrm{HH}}=\right.$ $12.5,=\mathrm{CH}), 6.58\left(\mathrm{t}, 1 \mathrm{H},{ }^{3} J_{\mathrm{HH}}=12.4,=\mathrm{CH}\right), 6.70\left(\mathrm{~d}, 1 \mathrm{H},{ }^{3} J_{\mathrm{HH}}=\right.$ 12.7, $=\mathrm{CH}), 7.41\left(\mathrm{t}, 1 \mathrm{H},{ }^{3} J_{\mathrm{HH}}=7.6\right.$, aromatic $), 7.46-7.54(\mathrm{~m}, 2 \mathrm{H}$, aromatic, $=\mathrm{CH}), 7.58\left(\mathrm{t}, 1 \mathrm{H},{ }^{3} \mathrm{~J}_{\mathrm{HH}}=7.9\right.$, aromatic $), 7.61-7.68(\mathrm{~m}$, $2 \mathrm{H}$, aromatic, $=\mathrm{CH}), 7.74\left(\mathrm{~d}, 2 \mathrm{H},{ }^{3} J_{\mathrm{HH}}=8.5\right.$, aromatic $), 7.96(\mathrm{t}, 1$ $\left.\mathrm{H},{ }^{3} \mathrm{~J}_{\mathrm{HH}}=14.4,=\mathrm{CH}\right), 7.99-8.06(\mathrm{~m}, 3 \mathrm{H}$, aromatic $), 8.23(\mathrm{~d}, 1 \mathrm{H}$, ${ }^{3} J_{\mathrm{HH}}=8.7$, aromatic). ${ }^{13} \mathrm{C}$ NMR spectrum (DMSO- $d_{6}, \delta$, ppm, $\mathrm{J} / \mathrm{Hz}): 22.5$ (s, $\left.1 \mathrm{C}, \mathrm{CH}_{2}\right), 26.3$ (s, $\left.1 \mathrm{C}, \mathrm{CH}_{2}\right), 26.7$ (s, $\left.2 \mathrm{C}, \mathrm{C}\left(\mathrm{CH}_{3}\right)_{2}\right)$,

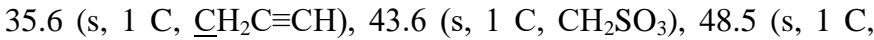
$\left.\underline{\mathrm{C}}\left(\mathrm{CH}_{3}\right)_{2}\right), 50.7\left(\mathrm{~s}, 1 \mathrm{C},+\mathrm{NCH}_{2}\right), 76.6(\mathrm{~s}, 1 \mathrm{C}, \mathrm{C} \equiv \underline{\mathrm{CH}}), 76.8(\mathrm{~s}, 1 \mathrm{C}$, $\underline{\mathrm{C}} \equiv \mathrm{CH}), 102.1,102.2,111.6$ (all s, $1 \mathrm{C}$, all $=\mathrm{CH}), 113.1,122.1$, 123.2 (all s, $1 \mathrm{C}$, all aromatic), 124.5 (s, $1 \mathrm{C},=\mathrm{CH}), 124.7,125.1$, 125.2, 127.5, 127.5, 128.1 (all s, $1 \mathrm{C}$, all aromatic), 128.1 (s, $1 \mathrm{C}$, $=\mathrm{CH}), 129.8,130.2,130.2,131.1,132.9,139.9($ all s, $1 \mathrm{C}$, all aromatic), 140.6 (s, $1 \mathrm{C}, \mathrm{N}-\mathrm{C}-\mathrm{S}$ ), 144.9 (s, $1 \mathrm{C}$, aromatic), 148.7, 154.1 (all s, $1 \mathrm{C}$, all $=\mathrm{CH}), 194.5(\mathrm{~s}, 1 \mathrm{C},+\mathrm{N}=\mathrm{C})$. HRMS-ESI: found $\mathrm{m} / \mathrm{z} 595.2083[\mathrm{M}+\mathrm{H}]$. Calculated for $\mathrm{C}_{35} \mathrm{H}_{34} \mathrm{~N}_{2} \mathrm{~S}_{2} \mathrm{O}_{3}{ }^{+}$ 595.2084. IR, $v / \mathrm{cm}^{-1}$ : $1052\left(\mathrm{SO}_{3}\right), 1389$ (N tret.), $2118(\mathrm{C} \equiv \mathrm{CH})$.

\subsection{Synthesis of daunorubicin azide (1)}

To a solution of daunorubicin hydrochloride $(50.0 \mathrm{mg}, 88.7$ mmol, 1 eq) in of $\mathrm{CH}_{2} \mathrm{Cl}_{2}(8 \mathrm{~mL})$ 4-azidobutyric acid oxysuccinimide ester (49.6 mg, $219.2 \mathrm{mmol}, 2.5 \mathrm{eq})$ and diisopropylethylamine ( $772 \mu \mathrm{L}, 4.4 \mathrm{mmol}, 50 \mathrm{eq})$ was added. The reaction mixture was stirred at $20^{\circ} \mathrm{C}$ for $10 \mathrm{~h}$ with control by TLC $\left(\mathrm{CH}_{2} \mathrm{Cl}_{2}-\mathrm{MeOH}, 10: 1\right)$ and HPLC (VDSpher $\mathrm{C} 8$ column, method: $20 \mathrm{~min} 25-95 \% \mathrm{MeCN}-\mathrm{H}_{2} \mathrm{O}$ with absorption detection at $260 \mathrm{~nm}$ ). Then solvent was evaporated. The residue was dissolved in $\mathrm{CH}_{2} \mathrm{Cl}_{2}$ and purified by silica gel column chromatography $\left(\mathrm{CH}_{2} \mathrm{Cl}_{2}-\right.$ $\mathrm{MeOH}, 50: 1 \mathrm{v} / \mathrm{v})$. Yield: $50 \mathrm{mg}(83 \%)$, red powder. ${ }^{1} \mathrm{H}$ NMR spectrum $\left(\mathrm{CDCl}_{3}, \delta\right.$, ppm, J/Hz): 1.25 (br. s., $\left.1 \mathrm{H}, \mathrm{CHO} \underline{\mathrm{H}}\right), 1.29$ (d, $\left.3 \mathrm{H},{ }^{3} \mathrm{~J}_{\mathrm{HH}}=6.5, \mathrm{CHC}_{3}\right), 1.76\left(\mathrm{~m}, 2 \mathrm{H}, \mathrm{CHC}_{2} \mathrm{CH}\right), 1.85(\mathrm{~m}, 2 \mathrm{H}$, $\left.\mathrm{CH}_{2} \mathrm{CH}_{2} \mathrm{CH}_{2}\right), 2.08\left(\mathrm{dd}, 1 \mathrm{H},{ }^{2} J_{H H}=14.98,{ }^{3} J_{H H}=3.37\right.$, $\mathrm{CHC}_{2} \mathrm{CH}$ ), $2.22\left(\mathrm{dt}, 2 \mathrm{H},{ }^{3} J_{H H}=7.40,1.77, \mathrm{CH}_{2} \mathrm{CH}_{2} \mathrm{CO}\right.$ ), 2.29 (d, $\left.1 \mathrm{H},{ }^{3} \mathrm{~J}_{\mathrm{HH}}=3.37, \mathrm{CHC}_{2} \mathrm{CH}\right), 2.41\left(\mathrm{~s}, 3 \mathrm{H}, \mathrm{CH}_{3} \mathrm{CO}\right), 2.82(\mathrm{~d}, 1 \mathrm{H}$, $\left.{ }^{2} J_{H H}=18.34, \mathrm{CC}_{2} \mathrm{C}\right), 3.18\left(\mathrm{~d}, 1 \mathrm{H},{ }^{2} J_{H H}=18.34, \mathrm{CC}_{2} \mathrm{C}\right), 3.64$ (br. s., $1 \mathrm{H}, \mathrm{C} \underline{\mathrm{HOH}}$ ), 3,80 (d, 2H, ${ }^{3} J_{H H}=5.69, \mathrm{~N}_{3} \underline{\mathrm{C}}_{2} \mathrm{CH}_{2}$ ), 4.04 (s, $\left.3 \mathrm{H}, \mathrm{CH}_{3} \mathrm{O}\right), 4.15$ (m, 1H, CHNH), $4.22\left(\mathrm{~m}, 1 \mathrm{H}, \mathrm{CH}_{3} \mathrm{CH}\right), 5.19$ (br. s., $1 \mathrm{H}, \mathrm{COH}), 5.47\left(\mathrm{~d}, 1 \mathrm{H},{ }^{3} J_{H H}=3.37, \mathrm{CHO}\right), 6.00\left(\mathrm{~d}, 1 \mathrm{H},{ }^{3} J_{H H}=\right.$ 8.31 , OCHO), $7.36\left(\mathrm{~d}, 1 \mathrm{H},{ }^{3} J_{H H}=8.38\right.$, aromatic), $7.76\left(\mathrm{t}, 1 \mathrm{H},{ }^{3} J_{H H}\right.$ $=8.38$, aromatic $), 7.99\left(\mathrm{~d}, 1 \mathrm{H},{ }^{3} J_{H H}=8.38\right.$, aromatic $), 13.20(\mathrm{~s}$, $1 \mathrm{H}, \mathrm{OH}), 13.95(\mathrm{~s}, 1 \mathrm{H}, \mathrm{OH}) .{ }^{13} \mathrm{C}$ NMR spectrum $\left(\mathrm{CDCl}_{3}, \delta, \mathrm{ppm}\right.$, $\mathrm{J} / \mathrm{Hz}): 16.3$ (s, $\left.\mathrm{CHCH}_{3}\right), 24.3\left(\mathrm{~s}, \mathrm{OC}^{-} \mathrm{H}_{3}\right), 24.5$ (s, $\left.\mathrm{CH}_{2} \underline{\mathrm{CH}}_{2} \mathrm{CH}_{2}\right)$, 29.5 (s, $\left.\mathrm{CHCH}_{2} \mathrm{CH}\right), 32.7$ (s, $\left.\mathrm{COCH}_{2} \mathrm{CH}_{2}\right), 32.9$ (s, $\left.\mathrm{CCH}_{2} \mathrm{COH}\right)$, 34.6 (s, $\left.\mathrm{CHCH}_{2} \mathrm{COH}\right), 44.9$ (s, $\left.\underline{\mathrm{CHNH}}\right), 50.3\left(\mathrm{~s}, \mathrm{~N}_{3} \mathrm{CH}_{2}\right), 56.2(\mathrm{~s}$, $\left.\mathrm{OC}_{3}\right)_{3}, 66.7$ (s, $\left.\mathrm{CH}_{3} \underline{\mathrm{CH}}\right), 69.1$ (s, $\left.\left(\mathrm{CH}_{2}\right) \mathrm{C} \underline{\mathrm{CHO}}\right), 69.6$ (s, $\left.\underline{\mathrm{CHOH}}\right)$, 76.2 (s, $\mathrm{CH}_{2} \mathrm{COH}$ ), 100.2 (s, OCHO), 110.8, 111.0, 118.0, 119.4, $120.3,133.5,134.0,135.0,135.3$, (all s., aromatic), 155.3, 156.0 (all s., aromatic, $\underline{\mathrm{COH}}$ ), 160.5 (s, aromatic, $\left.\mathrm{COCH}_{3}\right), 170.7$ (s, $\underline{\mathrm{CONH}}$ ), 186.1, 186.5 (all s., $\underline{\mathrm{CO}}), 211.8$ (s, $\left.\mathrm{HOC} \underline{\mathrm{C}}(\mathrm{O}) \mathrm{CH}_{3}\right)$. HRMS-ESI: found $\mathrm{m} / \mathrm{z} 639.2307[\mathrm{M}+\mathrm{H}]^{+}$. Calculated for $\mathrm{C}_{31} \mathrm{H}_{35} \mathrm{~N}_{4} \mathrm{NaO}_{11}+639.2297$.

\subsection{Synthesis of daunorubicin azide (2)}

Daunorubicin hydrochloride $(23.0 \mathrm{mg}, 40.7 \mathrm{mmol}, 1 \mathrm{eq})$ was treated by solution $O$-(2-(2-azidoethoxy)ethyl)hydroxylammonium chloride (30.9 $\mathrm{mg}, 114.1 \mathrm{mmol}, 2.8 \mathrm{eq})$ in DMSO $(2.5 \mathrm{~mL})$, then dry $\mathrm{Na}_{2} \mathrm{SO}_{4}(527 \mathrm{mg})$ was added. The reaction mixture was stirred at $20^{\circ} \mathrm{C}$ for $10 \mathrm{~h}$ with control by TLC $\left(\mathrm{CH}_{2} \mathrm{Cl}_{2}-\mathrm{MeOH}-\mathrm{AcOH}, 120: 40: 1\right)$ and HPLC (VDSpher C8 column, method: 20 min $25-95 \% \mathrm{MeCN}-\mathrm{H}_{2} \mathrm{O}$ with absorption detection at $260 \mathrm{~nm}$ ). Then the reaction mixture was freeze-dried. The residue was dissolved in $\mathrm{CH}_{2} \mathrm{Cl}_{2}$, filtered off and purified by silica gel column chromatography $\left(\mathrm{CH}_{2} \mathrm{Cl}_{2}-\mathrm{MeOH}, 3: 1 \mathrm{v} / \mathrm{v}\right)$. Yield: $26.1 \mathrm{mg}$ (82\%), red powder. ${ }^{1} \mathrm{H}$ NMR spectrum (DMSO- $d_{6}$, $\delta$, ppm, J/Hz): $1.16\left(\mathrm{~d}, 3 \mathrm{H},{ }^{3} J_{H H}=6.5, \mathrm{CHCH}_{3}\right), 1.71(\mathrm{~m}, 1 \mathrm{H}$, $\mathrm{CHCH}_{2} \mathrm{CHNH}_{2}$ ), 1.83 (s., $\left.3 \mathrm{H}, \mathrm{NCCH}_{3}\right) 1.88$ (m, $1 \mathrm{H}$, $\left.\mathrm{CHCH}_{2} \mathrm{CHNH}_{2}\right), 2.19\left(\mathrm{~m}, 1 \mathrm{H}, \mathrm{OCHCH}_{2} \mathrm{COH}\right), 2.28(\mathrm{~m}, 1 \mathrm{H}$, $\left.\mathrm{OCHC} \underline{\mathrm{H}}_{2} \mathrm{COH}\right) 2.85\left(\mathrm{~m}, 1 \mathrm{H}, \mathrm{HOCCC} \underline{\bar{H}}_{2} \mathrm{COH}\right), 3.05(\mathrm{~m}, 1 \mathrm{H}$, $\left.\mathrm{HOCCC} \underline{\mathrm{H}}_{2} \mathrm{COH}\right), 3.35$ (m, $\left.3 \mathrm{H}, \underline{\mathrm{CH}}_{2} \mathrm{~N}_{3}, \underline{\mathrm{CHNH}}_{2}\right), 3.39,3.40,3.43$, 3.48 (all m, $\left.\mathrm{CH}_{2} \mathrm{OC}_{2} \underline{\mathrm{C}}_{2} \mathrm{OCH}_{2}\right), 3.50\left(\mathrm{~m}, \mathrm{NOCH}_{2} \mathrm{CH}_{2}\right), 3.55$ (t, $\left.{ }^{3} J_{H H}=5,1, \mathrm{OCH}_{2} \mathrm{CH}_{2} \mathrm{~N}_{3}\right), 3.62(\mathrm{~m}, 1 \mathrm{H}, \mathrm{CHOH}), 3.94(\mathrm{~s}, 3 \mathrm{H}$, $\left.\mathrm{OC}_{3}\right), 4.02\left(\mathrm{~m}, 2 \mathrm{H}, \mathrm{NOCH}_{2}\right), 4.11\left(\mathrm{~m}, 1 \mathrm{H}, \mathrm{CHCH}_{3}\right), 4.90(\mathrm{~m}, 1$ $\left.\mathrm{H}, \mathrm{CC} \underline{\mathrm{H}}(\mathrm{O}) \mathrm{CH}_{2}\right), 5.28(\mathrm{~m}, 1 \mathrm{H}, \mathrm{OC} \underline{\mathrm{HO}}), 5.31$ (br. s., $1 \mathrm{H}$, $\mathrm{CH}_{2} \mathrm{C}(\mathrm{OH}) \mathrm{CN}$ ), 5.46 (br. s., $\left.1 \mathrm{H}, \mathrm{CHOH}\right), 7.57$ (br. s., $1 \mathrm{H}$, $\left.\mathrm{CHCOCH}_{3}\right), 7.82\left(\mathrm{~m}, 2 \mathrm{H}, \mathrm{CC} \underline{\mathrm{HC}} \underline{\mathrm{HCH}}\right.$, aromatic). ${ }^{13} \mathrm{C} \mathrm{NMR}$ spectrum (DMSO- $\left.d_{6}, \delta, \mathrm{ppm}, \mathrm{J} / \mathrm{Hz}\right): 10.2\left(\mathrm{~s}, \mathrm{NCCH}_{3}\right), 17.3(\mathrm{~s}$, $\left.\mathrm{CHCH}_{3}\right), 28.7$ (s, $\left.\mathrm{CHCH}_{2} \mathrm{CHNH}_{2}\right), 33.5$ (s, $\left.\mathrm{HOCCC} \underline{\mathrm{H}}_{2} \mathrm{COH}\right), 47.1$ $\left(\mathrm{s}, \underline{\mathrm{C}} \mathrm{HNH}_{2}\right), 50.4\left(\mathrm{~s}, \underline{\mathrm{C}} \mathrm{H}_{2} \mathrm{~N}_{3}\right), 57.0\left(\mathrm{~s}, \mathrm{OCH}_{3}\right), 6 \overline{6} .5(\mathrm{~s}, \underline{\mathrm{CHOH}})$, $66.7\left(\mathrm{~s}, \mathrm{CH}_{3} \underline{\mathrm{CH}}\right), 69.7\left(\mathrm{~s}, \underline{\mathrm{CH}_{2}} \mathrm{CH}_{2} \mathrm{~N}_{3}\right), 69.1,70.1,70.14,70.18$ (all $\left.\mathrm{s}, \quad \mathrm{CH}_{2} \mathrm{OCH}_{2} \mathrm{CH}_{2} \mathrm{OCH}_{2}\right), 71.6\left(\mathrm{~s}, \quad \mathrm{CCH}(\mathrm{O}) \mathrm{CH}_{2}\right), 71.8 \quad(\mathrm{~s}$, $\mathrm{CH}_{2} \mathrm{C}(\mathrm{OH}) \mathrm{CN}$ ), 73.0 (s, NOCH${ }_{2}$ ), 99.6 (s, OCHO), 110.9, 111.1 (all s, O=CECOH), $116.7 \quad\left(\mathrm{~s}, \quad \mathrm{O}=\mathrm{CCCOCH}_{3}\right), 120.0 \quad(\mathrm{~s}$, $\mathrm{O}=\mathrm{CC} \underline{\mathrm{CHCH}}$, aromatic), 120.4 (s, $\mathrm{CH} \underline{\mathrm{C}} \mathrm{HCOCH} \mathrm{H}_{3}$, aromatic), 135.1 (s, O=C대CH, aromatic), 135.9 (s, $\mathrm{HOCCCH}_{2} \mathrm{COH}$, aromatic), 136.3 (s, $\mathrm{HOCCC}(\mathrm{O}) \mathrm{CH}_{2}$, aromatic), 136.6 (s, $\mathrm{CHCHCH}$, aromatic), 154.9 (s, $\mathrm{HOCCCH}_{2} \mathrm{COH}$, aromatic), 156.8 (s, $\mathrm{HOCCC}(\mathrm{O}) \mathrm{CH}_{2}$, aromatic), $160.0 \quad(\mathrm{~s}, \underline{\mathrm{C}}=\mathrm{NO}$ ), 161.1 (s, $\mathrm{COCH}_{3}$, aromatic), 186.7 (s, $2 \mathrm{C}, \mathrm{C}=\mathrm{O}$, aromatic). HRMS-ESI: found $\mathrm{m} / \mathrm{z} 744.3078[\mathrm{M}+\mathrm{H}]^{+}$. Calculated for $\mathrm{C}_{35} \mathrm{H}_{45} \mathrm{~N}_{5} \mathrm{O}_{13}{ }^{+}$ 744.3087

\subsection{General procedure for the synthesis of conjugates $\mathbf{6} \boldsymbol{a}-\boldsymbol{b}$ and $7 a-b$}

A solution of daunorubicin azide 1 or $2(8 \mu \mathrm{mol})$ of $\mathrm{MeOH}(1$ $\mathrm{mL}$ ) was treated by tricarbocyanine dye $\mathbf{5 a}$ or $\mathbf{5 b}$, a solution of a dye $(7.4 \mathrm{umol})$ of DMSO $(1 \mathrm{~mL})$. Then ascorbic acid $(0.28 \mathrm{M}$ aqueous solution (4 mmol) and $\mathrm{CuSO}_{4-}$ tris(benzyltriazolylmethyl)amine, 1:1 (0.01 M solution of in 55\% DMSO) were added, the reaction mixture was stirred under argon atmosphere for $10 \mathrm{~h}$ with control by TLC. After consumption of starting materials the reaction mixture was concentrated in vacuo and freeze-dried. The residue was purified by silica gel column chromatography.

\subsubsection{Conjugate $6 a$}

Yield: $6.4 \mathrm{mg}$ (69\%), green powder. TLC: $\mathrm{CH}_{2} \mathrm{Cl}_{2}-\mathrm{MeOH}$ 10:1 (v/v). Eluent for column chromatography: $\mathrm{CH}_{2} \mathrm{Cl}_{2}-\mathrm{MeOH}$ 15:1 (v/v). HRMS-ESI: found $\mathrm{m} / \mathrm{z} 1181.5043[\mathrm{M}]^{+}$. Calculated for $\mathrm{C}_{68} \mathrm{H}_{73} \mathrm{~N}_{6} \mathrm{O}_{11} \mathrm{~S}^{+} \mathrm{M} 1181.5053$.

\subsubsection{Conjugate $\mathbf{6} \boldsymbol{b}$}

Yield: $9.0 \mathrm{mg}$ (93\%), green powder. TLC: $\mathrm{CH}_{2} \mathrm{Cl}_{2}-\mathrm{MeOH}$, 10:1 (v/v). Eluent for column chromatography: $\mathrm{CH}_{2} \mathrm{Cl}_{2}-\mathrm{MeOH}$ 5:1 (v/v). HRMS-ESI: found m/z $1255.4106[\mathrm{M}]^{+}$. Calculated for $\mathrm{C}_{66} \mathrm{H}_{68} \mathrm{~N}_{6} \mathrm{O}_{14} \mathrm{~S}_{2} \mathrm{Na}^{+} 1255.4127$

\subsubsection{Conjugate $7 a$}

Yield: $10.4 \mathrm{mg}$ (90\%), green powder. TLC: $\mathrm{CH}_{2} \mathrm{Cl}_{2}-\mathrm{MeOH}$, 5:1 (v/v). Eluent for column chromatography: $\mathrm{CH}_{2} \mathrm{Cl}_{2}-\mathrm{MeOH}$ 5:1 (v/v). HRMS-ESI: found $\mathrm{m} / \mathrm{z} 1286.5811[\mathrm{M}]^{+}$. Calculated for $\mathrm{C}_{72} \mathrm{H}_{84} \mathrm{~N}_{7} \mathrm{O}_{13} \mathrm{~S}^{+} 1286.5842$.

\subsubsection{Conjugate $7 \boldsymbol{b}$}


Yield: $4.6 \mathrm{mg}$ (50\%), green powder. TLC: $\mathrm{CH}_{2} \mathrm{Cl}_{2}-\mathrm{MeOH}, 5: 1$ (v/v). Eluent for column chromatography: $\mathrm{CH}_{2} \mathrm{Cl}_{2}-\mathrm{MeOH}$ 4:1 (v/v). HRMS-ESI: found $\mathrm{m} / \mathrm{z} 1338.5096[\mathrm{M}]^{+}$. Calculated for $\mathrm{C}_{70} \mathrm{H}_{80} \mathrm{~N}_{7} \mathrm{O}_{16} \mathrm{~S}_{2}{ }^{+} 1338.5097$.

\subsection{MTT-assay}

The cytotoxic activity of compounds was assessed in vitro using the MTT assay against human tumor cell lines, such as human colon cancer cells HCT116. The cells were obtained from American Type Culture Collection, USA. Cells were cultivated in Dulbecco modified Eagle's medium supplemented with $10 \%$ fetal bowine serum (HyClone, Logan, UT), 2 mM L-glutamine, 100 $\mathrm{U} / \mathrm{mL}$ penicillin, and $100 \mu \mathrm{g} / \mathrm{mL}$ streptomycin at $37^{\circ} \mathrm{C}$ in a humidified $\mathrm{CO}_{2}$-controlled atmosphere. Cells in logarithmic phase of growth were used in the experiments. To prevent mycoplasma infection, the drug Mycokill (GE, USA) was used. Before the start of the experiments, at least three passages were carried out on a medium free from antimycoplasma drug. The samples were used as $10 \mathrm{mM}$ stock solutions in DMSO. Samples of the compounds were stored at $+4^{\circ} \mathrm{C}$, thawed at room temperature immediately before use. Cells were plated in 96-well plates (NUNC, USA, $5 \cdot 10^{3}$ cells in $190 \mu \mathrm{L}$ culture medium per well). After $24 \mathrm{~h}$ of incubation at $37^{\circ} \mathrm{C}\left(5 \% \mathrm{CO}_{2}\right.$, in a humidified atmosphere), $10 \mu \mathrm{l}$ of the solutions of test compounds to final concentrations $0.1-50$ $\mu \mathrm{mol} / \mathrm{L}$ were added to the cells. The cells without substances served as an intact control. The cells were incubated for $72 \mathrm{~h}$ at $37^{\circ} \mathrm{C}, 5 \% \mathrm{CO}_{2}$, in a humidified atmosphere; $2 \mathrm{~h}$ before the end of incubation, $20 \mu \mathrm{L}$ of aqueous solution of MTT $(5 \mathrm{mg} / \mathrm{mL}$, PanEko, Russia) was added to the wells. After the end of the incubation, the culture medium was removed, the cells were resuspended in 100 $\mu \mathrm{L}$ of DMSO, and the optical density of the solution was measured with Multiscan FC plate spectrophotometer (Thermo Scientific, USA) at $571 \mathrm{~nm}$. The percentage of survived cells survived was calculated as a quotient from the division of the average optical density in the wells after incubation with a given dose to the average optical density of the control wells. Five independent experiments were carried out for each concentration; the results are statistically processed. Standard deviations did not exceed $10 \%$.

4.10. Laser scanning confocal microscopy was used to visualize colocalization, the morphology of mitochondria and to monitor the integrity of the plasma membrane upon treatment with synthesized compounds. The HCT116 cells were plated on $35 \mathrm{~mm}$ dishes with the glass bottom (SPL Life Sci., Korea) and incubated for $72 \mathrm{~h}$ at $37^{\circ} \mathrm{C}, 5 \% \mathrm{CO}_{2}$ to reach $50 \%$ confluence. Then the cells were incubated with synthesized compounds for up to $24 \mathrm{~h}$ followed by washing with PBS. For labeling mitochondria, nuclei and lysosomes, dihydrorhodamine 123 (DHR 123; (476 $\mathrm{nm} / 509-582 \mathrm{~nm})$, Hoechst $33342(405 \mathrm{~nm} / 410-500 \mathrm{~nm})$ and Lysotracker Green (476 nm/509-582 nm), respectively, were used as recommended by the manufacturer (Thermo Fisher Sci.). Dyes flare into the DHR123/LysotrackerGreen channels was excluded by regulation of the laser power and detector gain in samples with sequential staining. Images were analyzed on a laser scanning confocal microscope Leica TCS SPE 5 with LAS AF software (Leica Microsystems GmbH, Germany).

\section{Acknowledgments}

The research was supported in part by Russian Science Foundation (project 20-15-00361). K.A.S. was supported by Russian Foundation for Basic Research (project 20-34-90125).

\section{References and notes}

(3) Sun, C.; Du, W.; Wang, B.; Dong, B.; Wang, B. Research Progress of Near-Infrared Fluorescence Probes Based on Indole Heptamethine Cyanine Dyes in Vivo and in Vitro. BMC Chemistry 2020, 14 (1), 21. https://doi.org/10.1186/s13065-020-00677-3.

(4) Deng, G.; Li, S.; Sun, Z.; Li, W.; Zhou, L.; Zhang, J.; Gong, P.; Cai, L. Near-Infrared Fluorescence Imaging in the Largely Unexplored Window of 900-1,000 Nm. Theranostics 2018, 8 (15), 4116-4128. https://doi.org/10.7150/thno.26539.

(5) Lange, N.; Szlasa, W.; Saczko, J.; Chwiłkowska, A. Potential of Cyanine Derived Dyes in Photodynamic Therapy. Pharmaceutics 2021, 13 (6), 818. https://doi.org/10.3390/pharmaceutics 13060818 .

(6) Yang, X.; Bai, J.; Qian, Y. The Investigation of Unique WaterSoluble Heptamethine Cyanine Dye for Use as NIR Photosensitizer in Photodynamic Therapy of Cancer Cells. Spectrochimica Acta Part A: Molecular and Biomolecular Spectroscopy 2020, 228, 117702. https://doi.org/10.1016/j.saa.2019.117702

(7) Atchison, J.; Kamila, S.; Nesbitt, H.; Logan, K. A.; Nicholas, D. M.; Fowley, C.; Davis, J.; Callan, B.; McHale, A. P.; Callan, J. F. Iodinated Cyanine Dyes: A New Class of Sensitisers for Use in NIR Activated Photodynamic Therapy (PDT). Chem. Commun. 2017, 53 (12), 2009-2012. https://doi.org/10.1039/C6CC09624G.

(8) Wang, A.; Mao, Q.; Zhao, M.; Ye, S.; Fang, J.; Cui, C.; Zhao, Y. Zhang, Y.; Zhang, Y.; Zhou, F.; Shi, H. PH/Reduction Dual Stimuli-Triggered Self-Assembly of NIR Theranostic Probes for Enhanced Dual-Modal Imaging and Photothermal Therapy of Tumors. Anal. Chem. 2020, 92 (24), 16113-16121. https://doi.org/10.1021/acs.analchem.0c03800.

(9) Leitão, M. M.; de Melo-Diogo, D.; Alves, C. G.; Lima-Sousa, R.; Correia, I. J. Prototypic Heptamethine Cyanine Incorporating Nanomaterials for Cancer Phototheragnostic. Adv. Healthcare Mater. 2020, 9 (6), 1901665 https://doi.org/10.1002/adhm.201901665.

(10) Hu, D.; Pan, M.; Yang, Y.; Sun, A.; Chen, Y.; Yuan, L.; Huang, K. Qu, Y.; He, C.; Wei, Q.; Qian, Z. Trimodal Sono/Photoinduced Focal Therapy for Localized Prostate Cancer: Single-Drug-Based Nanosensitizer under Dual-Activation. Advanced Functional Materials 2021, $n / a(\mathrm{n} / \mathrm{a}), 2104473$.

https://doi.org/10.1002/adfm.202104473.

(11) Black, C. E.; Zhou, E.; DeAngelo, C.; Asante, I.; Yang, R.; Petasis, N. A.; Louie, S. G.; Humayun, M. Cyanine Nanocage Activated by Near-IR Light for the Targeted Delivery of Cyclosporine A to Traumatic Brain Injury Sites. Mol. Pharmaceutics 2020, 17 (12), 4499-4509. https://doi.org/10.1021/acs.molpharmaceut.0c00589.

(12) Zhang, Y.; Yan, C.; Zheng, Q.; Jia, Q.; Wang, Z.; Shi, P.; Guo, Z Harnessing Hypoxia-Dependent Cyanine Photocages for In Vivo Precision Drug Release. Angew. Chem. Int. Ed. 2021, 60 (17), 9553-9561. https://doi.org/10.1002/anie.202017349.

(13) Bokan, M.; Gellerman, G.; Patsenker, L. D. Drug Delivery Platform Comprising Long-Wavelength Fluorogenic Phenolo-Cyanine Dye for Real-Time Monitoring of Drug Release. Dyes and Pigments 2019, 171, 107703. https://doi.org/10.1016/j.dyepig.2019.107703.

(14) Li, Y.; Zhou, Y.; Yue, X.; Dai, Z. Cyanine Conjugates in Cancer Theranostics. Bioactive Materials 2021, 6 (3), 794-809. https://doi.org/10.1016/j.bioactmat.2020.09.009.

(15) Cooper, E.; Choi, P. J.; Denny, W. A.; Jose, J.; Dragunow, M.; Park, T. I.-H. The Use of Heptamethine Cyanine Dyes as DrugConjugate Systems in the Treatment of Primary and Metastatic Brain Tumors. Front Oncol 2021, 11, 654921. https://doi.org/10.3389/fonc.2021.654921.

(16) Shi, C.; Wu, J. B.; Chu, G. C.-Y.; Li, Q.; Wang, R.; Zhang, C.; Zhang, Y.; Kim, H. L.; Zhau, H. E.; Pan, D.; Chung, L. W. K. Heptamethine Carbocyanine Dye-Mediated near-Infrared Imaging of Canine and Human Cancers through the HIF-1 $\alpha /$ OATPs Signaling Axis. Oncotarget 2014, 5 (20), 10114-10126. https://doi.org/10.18632/oncotarget.2464.

(17) Nödling, A. R.; Mills, E. M.; Li, X.; Cardella, D.; Sayers, E. J.; Wu, S.-H.; Jones, A. T.; Luk, L. Y. P.; Tsai, Y.-H. Cyanine Dye Mediated Mitochondrial Targeting Enhances the Anti-Cance Activity of Small-Molecule Cargoes. Chem. Commun. 2020, 56 (34), 4672-4675. https://doi.org/10.1039/C9CC07931A. 
(18) Usama, S. M.; Lin, C.-M.; Burgess, K. On the Mechanisms of Uptake of Tumor-Seeking Cyanine Dyes. Bioconjugate Chem. 2018, 29 (11), 3886-3895. https://doi.org/10.1021/acs.bioconjchem.8b00708.

(19) Choi, P. J.; Park, T. I.; Cooper, E.; Dragunow, M.; Denny, W. A.; Jose, J. Heptamethine Cyanine Dye Mediated Drug Delivery: Hype or Hope. Bioconjugate Chem. 2020, 31 (7), 1724-1739. https://doi.org/10.1021/acs.bioconjchem.0c00302.

(20) Cooper, E.; Choi, P. J.; Schweder, P.; Mee, E.; Turner, C.; Faull, R.; Denny, W. A.; Dragunow, M.; I-H. Park, T.; Jose, J. Cytoprotective Agent Troxipide-Cyanine Dye Conjugate with Cytotoxic and Antiproliferative Activity in Patient-Derived Glioblastoma Cell Lines. Bioorganic \& Medicinal Chemistry Letters 2021, 50, 128336. https://doi.org/10.1016/j.bmcl.2021.128336.

(21) Zhao, Y.; Zhang, H.; Wu, P.; Tan, D.; Zhao, Y.; Zhang, C.; Wang, J.; Bai, B.; An, J.; Shi, C. Mediated Imaging and Improved Targeting of Farnesylthiosalicylic Acid Delivery for Pancreatic Cancer via Conjugation with Near-Infrared Fluorescence Heptamethine Carbocyanine Dye. ACS Appl. Bio Mater. 2020, 3 (2), 1129-1138. https://doi.org/10.1021/acsabm.9b01068.

(22) Mrdenovic, S.; Zhang, Y.; Wang, R.; Yin, L.; Chu, G. C.; Yin, L.; Lewis, M.; Heffer, M.; Zhau, H. E.; Chung, L. W. K. Targeting Burkitt Lymphoma with a Tumor Cell-Specific Heptamethine Carbocyanine-cisplatin Conjugate. Cancer 2019, 125 (13), 22222232. https://doi.org/10.1002/cncr.32033.

(23) Zheng, Y.; Lan, T.; Wei, D.; Zhang, G.; Hou, G.; Yuan, J.; Yan, F.; Wang, F.; Meng, P.; Yang, X.; Chen, G.; Zhu, Z.; Lu, Z.; He, W.; Yuan, J. Coupling the Near-Infrared Fluorescent Dye IR-780 with Cabazitaxel Makes Renal Cell Carcinoma Chemotherapy Possible. Biomedicine \& Pharmacotherapy 2019, 116, 109001. https://doi.org/10.1016/j.biopha.2019.109001.

(24) Yang, X.; Hou, Z.; Wang, D.; Mou, Y.; Guo, C. Design, Synthesis and Biological Evaluation of Novel Heptamethine Cyanine DyeErlotinib Conjugates as Antitumor Agents. Bioorganic \& Medicinal Chemistry Letters 2020, 30 (23), 127557. https://doi.org/10.1016/j.bmcl.2020.127557.

(25) Guan, Y.; Zhang, Y.; Zou, J.; Huang, L.-P.; Chordia, M. D.; Yue, W.; Wu, J.-J.; Pan, D.-F. Synthesis and Biological Evaluation of Genistein-IR783 Conjugate: Cancer Cell Targeted Delivery in MCF-7 for Superior Anti-Cancer Therapy. Molecules 2019, 24 (22), 4120. https://doi.org/10.3390/molecules24224120.

(26) Etrych, T.; Daumová, L.; Pokorná, E.; Tušková, D.; Lidický, O.; Kolářová, V.; Pankrác, J.; Šefc, L.; Chytil, P.; Klener, P. Effective Doxorubicin-Based Nano-Therapeutics for Simultaneous Malignant Lymphoma Treatment and Lymphoma Growth Imaging. Journal of Controlled Release 2018, 289, 44-55.

https://doi.org/10.1016/j.jconrel.2018.09.018.

(27) Nadas, J.; Sun, D. Anthracyclines as Effective Anticancer Drugs. Expert Opinion on Drug Discovery 2006, 1 (6), 549-568. https://doi.org/10.1517/17460441.1.6.549.

(28) McGowan John V; Robin Chung; Angshuman Maulik; Izabela Piotrowska; J Malcolm Walker; Derek M Yellon. Anthracycline Chemotherapy and Cardiotoxicity. Cardiovasc Drugs Ther 31 (1), 63-75. https://doi.org/10.1007/s10557-016-6711-0.

(29) Bhagat, A.; Kleinerman, E. S. Anthracycline-Induced Cardiotoxicity: Causes, Mechanisms, and Prevention. In Current Advances in Osteosarcoma : Clinical Perspectives: Past, Present and Future; Kleinerman, E. S., Gorlick, R., Eds.; Advances in Experimental Medicine and Biology; Springer International Publishing: Cham, 2020; pp 181-192. https://doi.org/10.1007/9783-030-43032-0_15.

(30) O’Brien, M. E. R.; Wigler, N.; Inbar, M.; Rosso, R.; Grischke, E.; Santoro, A.; Catane, R.; Kieback, D. G.; Tomczak, P.; Ackland, S. P.; Orlandi, F.; Mellars, L.; Alland, L.; Tendler, C. Reduced Cardiotoxicity and Comparable Efficacy in a Phase IIItrial of Pegylated Liposomal Doxorubicin $\mathrm{HCl}\left(\mathrm{CAELYX} \mathrm{X}^{\mathrm{TM}} /\right.$ Doxil $\left.{ }^{\circledR}\right)$ versus Conventional Doxorubicin Forfirst-Line Treatment of
Metastatic Breast Cancer. Annals Oncol. 2004, 15 (3), 440-449. https://doi.org/10.1093/annonc/mdh097.

(31) Piorecka, K.; Kurjata, J.; Stanczyk, M.; Stanczyk, W. A. Synthetic Routes to Nanomaterials Containing Anthracyclines: Noncovalent Systems. Biomater. Sci. 2018, 6 (10), 2552-2565. https://doi.org/10.1039/C8BM00739J.

(32) Haldón, E.; Nicasio, M. C.; Pérez, P. J. Copper-Catalysed AzideAlkyne Cycloadditions (CuAAC): An Update. Org. Biomol. Chem. 2015, 13 (37), 9528-9550. https://doi.org/10.1039/C5OB01457C.

(33) Priebe, W. Mechanism of Action-Governed Design of Anthacycline Antibiotics: A “Turn-Off/Turn-On” Approach. Curr. Pharm. Des. 1995, 1 (1), 51-68.

(34) Effenberger, K.; Breyer, S.; Schobert, R. Modulation of Doxorubicin Activity in Cancer Cells by Conjugation with Fatty Acyl and Terpenyl Hydrazones. Eur. J. Med. Chem. 2010, 45 (5), 1947-1954. https://doi.org/10.1016/j.ejmech.2010.01.037.

(35) Korolev, A. M.; Lazhko, É. I.; Preobrazhenskaya, M. N.; Bal'zarini, Ya.; De Klerk, É. Amides of Anthracycline Antibiotics and NCarboxymethylascorbigen. Pharm Chem J 1991, 25 (11), 805-808. https://doi.org/10.1007/BF00767264.

(36) Sapozhnikova, K. A.; Misyurin, A. V.; Pestov, N. B.; Meleshkina, E. G.; Oreshkov, S. D.; Ganzhula, E. P.; Mikhailova, A. S.; Korshun, V. A.; Misyurin, V. A.; Brylev, V. A. Detection of PRAME Protein on the Surface of Melanoma Cells Using a Fluorescently Labeled Monoclonal Antibody. Rus. J. Bioorg. Chem. 2021. https://doi.org/10.1134/S1068162021050332.

(37) Kumar, V.; Remers, W. A.; Bradner, W. T. Preparation and Antitumor Activity of Olivomycin A Analogs. J. Med. Chem. 1980, 23 (4), 376-379. https://doi.org/10.1021/jm00178a006.

(38) Tyler, A. R.; Okoh, A. O.; Lawrence, C. L.; Jones, V. C.; Moffatt, C.; Smith, R. B. N-Alkylated 2,3,3-Trimethylindolenines and 2Methylbenzothiazoles. Potential Lead Compounds in the Fight against Saccharomyces Cerevisiae Infections. European Journal of Medicinal Chemistry 2013, 64, 222-227.

https://doi.org/10.1016/j.ejmech.2013.03.031.

(39) Cheng, W.-C.; Kurth, M. J. The Zincke Reaction. a Review.

Organic Preparations and Procedures International 2002, 34 (6), 585-608. https://doi.org/10.1080/00304940209355784.

(40) Davis, P. D. Discrete Peg Based Dyes. WO2015038579A1, March $19,2015$.

(41) Zhan, W.; Barnhill, H. N.; Sivakumar, K.; Tian, H.; Wang, Q. Synthesis of Hemicyanine Dyes for 'Click' Bioconjugation. Tetrahedron Letters 2005, 46 (10), 1691-1695. https://doi.org/10.1016/j.tetlet.2005.01.066.

(42) Henary, M.; Pannu, V.; Owens, E. A.; Aneja, R. Near Infrared Active Heptacyanine Dyes with Unique Cancer-Imaging and Cytotoxic Properties. Bioorganic \& Medicinal Chemistry Letters 2012, 22 (2), 1242-1246. https://doi.org/10.1016/j.bmcl.2011.11.070.

(43) Sunwoo, K.; Won, M.; Ko, K.-P.; Choi, M.; Arambula, J. F.; Chi, S.-G.; Sessler, J. L.; Verwilst, P.; Kim, J. S. Mitochondrial Relocation of a Common Synthetic Antibiotic: A Non-Genotoxic Approach to Cancer Therapy. Chem 2020, 6 (6), 1408-1419. https://doi.org/10.1016/j.chempr.2020.03.004.

(44) Lv, Q.; Yang, X.; Wang, M.; Yang, J.; Qin, Z.; Kan, Q.; Zhang, H.; Wang, Y.; Wang, D.; He, Z. Mitochondria-Targeted Prostate Cancer Therapy Using a near-Infrared Fluorescence DyeMonoamine Oxidase A Inhibitor Conjugate. Journal of Controlled Release 2018, 279, 234-242.

https://doi.org/10.1016/j.jconrel.2018.04.038.

(45) Mizrahi, D. M.; Ziv-Polat, O.; Perlstein, B.; Gluz, E.; Margel, S. Synthesis, Fluorescence and Biodistribution of a Bone-Targeted near-Infrared Conjugate. European Journal of Medicinal Chemistry 2011, 46 (10), 5175-5183.

https://doi.org/10.1016/j.ejmech.2011.08.040. 